\title{
Decay score: a guide to the immunoreactivity of human pancreatic islets in autopsy specimen
}

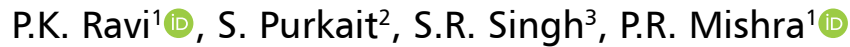 \\ 'Department of Anatomy, All India Institute of Medical Sciences, Bhubaneswar, Odisha, India \\ 2Department of Pathology, All India Institute of Medical Sciences, Bhubaneswar, Odisha, India \\ ${ }^{3}$ Department of Forensic Medicine and Toxicology, All India Institute of Medical Sciences, Bhubaneswar, Odisha, India
}

[Received: 24 November 2020; Accepted: 6 January 2021; Early publication date: 22 January 2021]

Background: The pancreas is an exo-endocrine organ that undergoes rapid autolysis soon after death, which limits its utility in academics and research. The timeline of autolytic changes of pancreatic islets and its immunoreactivity is limited in the literature. Decay score has been used to grade the autolytic changes in organs like the brain, lung and liver. However, reports are not available in the pancreas/pancreatic islets. Knowledge regarding the decay score may be used as a torchbearer for the immunoreactivity of human pancreatic islets in autopsy cases. The present study is aimed to provide an optimal cut-off time based on the decay score before which pancreatic specimens should be collected for the purpose of immunohistochemical studies (IHC) of pancreatic islets.

Materials and methods: Serial sections of 20 adult human pancreases obtained from the autopsy were subjected to haematoxylin and eosin (H\&E) and immunohistochemical staining. Autolytic changes of pancreatic islets were graded by using decay score in H\&E sections, which was compared with the results of the immunohistochemical reactivity of pancreatic islets in IHC sections.

Results and Conclusions: Pancreatic islets immunoreactivity was found to be well preserved in the samples collected early within 9 hours with a decay score of less than 1.4. There was an inverse relation of decay score and immunoreactivity of pancreatic islets. The decay score of less than 1.4 has better-preserved immunoreactivity than having more than 1.4. This knowledge will help researchers working in the field of the endocrine pancreas. (Folia Morphol 2022; 81, 1: 101-106)

Key words: autolysis, pancreatic islets, immunohistochemistry, decay score, human pancreas

\section{INTRODUCTION}

Brain-dead organ donors and autopsy are the two major sources of whole human pancreatic tissue for academics and research $[8,10,15,17]$. The availability of brain-dead donor pancreas is limited in developing countries. Thus, the autopsy is the only source of the whole pancreatic tissue, yet its utility is limited by the appearance of early autolytic changes following death $[4,6,13]$. Well-preserved micro-architecture and immunogenicity are the integral part of any histological and immunohistochemical studies (IHC) on pancreas, including islets. A review of studies on the human pancreas did not reveal any standardised cut off time following death within which the specimen 
Table 1. Information about the subjects

\begin{tabular}{lcccc}
\hline Case no. & Age [years] & Gender & Body mass index $\left[\mathbf{k g} / \mathbf{m}^{2}\right]$ & Time since death $[\mathbf{h}]$ \\
\hline 1 & 45 & Male & 29.30 & 13.5 \\
2 & 52 & Male & 28.73 & 4.5 \\
3 & 28 & Male & 21.10 & 9 \\
4 & 60 & Male & 22.20 & 7.5 \\
5 & 43 & Female & 18.49 & 7.25 \\
6 & 30 & Female & 20.57 & 8.5 \\
7 & Female & 20.89 & 13 \\
8 & 34 & Female & 22.22 & 10 \\
9 & 34 & Male & 24.28 & 5 \\
10 & 35 & Male & 18.33 & 5.25 \\
11 & 75 & Male & 19.08 & 9 \\
12 & 45 & Male & 19.77 & 12 \\
13 & 27 & Male & 28.48 & 7 \\
14 & 25 & Male & 21.47 & 5.5 \\
15 & 62 & Female & 21.32 & 4.5 \\
16 & 45 & Male & 25.79 & 7.5 \\
17 & 28 & Female & 23.61 & 7.25 \\
18 & 23 & Female & 33.25 & 12 \\
19 & 42 & Female & 18.92 & 10.5
\end{tabular}

has to be collected for IHC of pancreatic islets. Decay score has been used to grade the autolytic changes in organs like the brain, lung and liver. However, decay score grading of autolytic changes is not performed in the pancreas/pancreatic islets. The present study is aimed to provide an optimal cut-off time based on the decay score before which pancreatic specimens should be collected from autopsy for the purpose of IHC of pancreatic islets.

\section{MATERIALS AND METHODS}

Pancreas collection:

A total of 20 autopsy specimens of the adult human (age range 23-75 years) pancreas were collected after the approval of the Institutional Ethical Committee (vide approval No. IEC/AIIMS BBSR/PG Thesis/2017-18/22). The cause of death, time since death and existence of other co-morbid conditions were documented (Table 1). In most of the cases, the cause of death was road traffic accident and none of the cases were associated with chronic diseases like diabetes. Pancreatic specimens with congenital anomalies or showing gross abnormalities like a cyst, tumour was excluded.
The incision was made over the constricted neck region of the pancreas (in relation to portal vein) to divide the head from the rest of the pancreas. Then the rest of the pancreas was divided into two equal parts (body and tail) as described in the previous studies [3,12]. After overnight fixation in 10\% neutral buffered formalin solution, the whole coronal section and horizontal section of the pancreatic head, body and tail were again subdivided into small sub-blocks (approximately 40 sub-blocks were obtained from each pancreas). All the well-labelled sub-blocks were fixed in $10 \%$ neutral buffered formalin followed by automated tissue processing by Leica automated tissue processor. Sections from the first tissue block of each part of the pancreas (one each from head, body and tail region; first coronal section) were utilized for this study, the rest of the paraffin-embedded pancreatic tissue blocks were preserved for further studies. Serial sections from the selected block were stained with haematoxylin and eosin (H\&E) and immunohistochemically with anti-synaptophysin and anti-insulin antibodies. Cases showing features of occult pancreatitis in H\&E staining were also excluded and no IHC was done. 


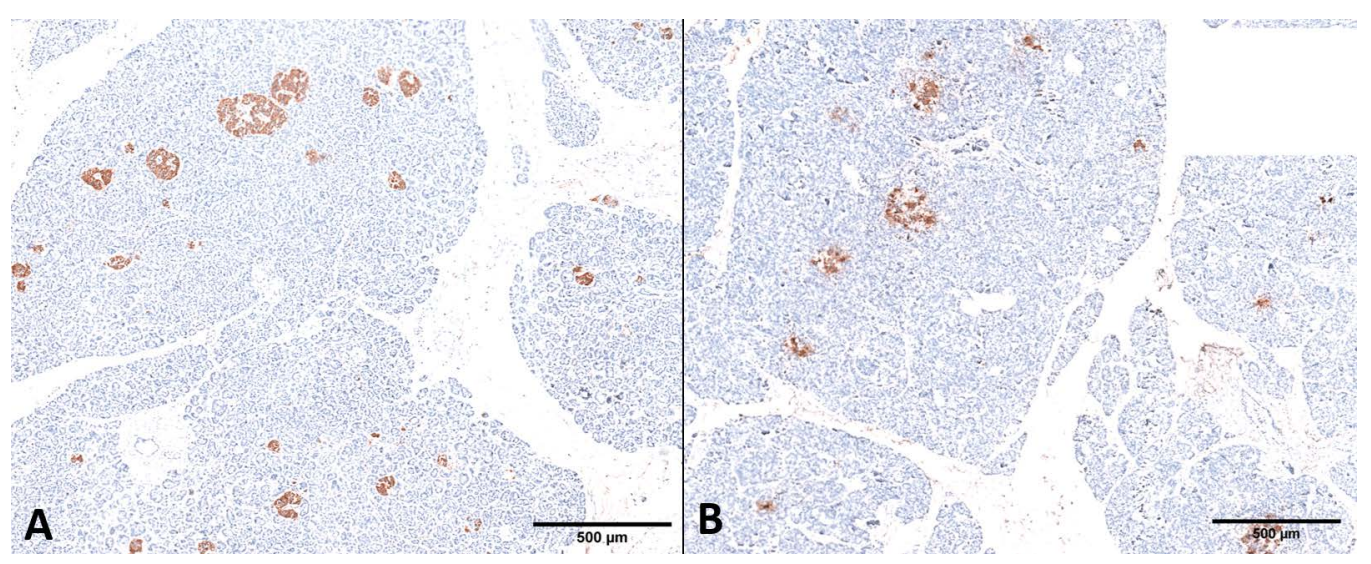

Figure 1. Photographs of immunohistochemistry slides showing sharp and strong positivity $(\mathbf{A})$ in the islets with decay score of less than 1.4; B. The diffused immunoreactivity.

\section{Immunohistochemistry}

Immunohistochemical studies were done on two consecutive $4 \mu \mathrm{m}$ thick paraffin sections. Antigen retrieval was done with citrate buffer by using the heat antigen retrieval method under pressure. Serial sections of each block were stained using a rabbit monoclonal anti-synaptophysin antibody (1:300) (PathnSitu, Livermore, California) (45 min incubation) for identifying islets and rabbit monoclonal anti-insulin (1:200) (PathnSitu, Livermore, California) (45 min incubation) for identifying beta-cells. The primary antibody was detected by a secondary antibody labelled with Horseradish peroxidase (HRP) and DAB (3,3-diaminobenzidine) chromogen (DAKO, Carpinteria, CA). Immunohistochemical positivity was assessed under $40 \times$ in a bright-field microscope (Olympus BX43 microscope) based on the rate of positivity and its sharpness (Fig. 1).

\section{Grading of autolysis}

All the H\&E slides were screened at lower power to identify the areas which showing maximum autolytic changes. Those identified areas were examined under a $40 \times$ bright field microscope (Olympus BX43 microscope). Five high power fields from each slide were used for the assessment of autolytic changes based on the cellular and nuclear architecture: normal nucleus (grade 1), pyknosis and minimal karyorrhexis (grade 2), prominent karyorrhexis (grade 3), no nucleus visible (grade 4) and no cell visible (grade 5) [9]. Autolytic changes will be heterogeneous even in single islets; i.e. cells within single islets will show different grades of nuclear changes. To maintain the uniform standard of scoring the autolytic changes we adapted the decay score for each high-power field from previous literature [9]. The decay score is calculated by multiplying the percentage of cells with a certain grade of nuclear changes by the value of grades (1-5) and summing these values. For example, within a single islet, $25 \%$ of cells showing features of grade 1 (normal), $50 \%$ of cells with grade 2 and $25 \%$ of cells with grade 3 autolytic changes, the overall decay score was calculated as $(0.25 \times 1)+$ $+(0.5 \times 2)+(0.25 \times 3)=2.0$. The final decay score of each slide will be calculated from the mean of five high-power fields. Decay score grading was done by three independent observers in different time period, the average score of the three observers was taken as the final score.

\section{Statistical analysis}

Data were summarised and expressed as mean \pm standard error of mean (SEM). One-way ANOVA was used to compare the data between more than two groups. Paired ' $\mathrm{t}$ ' test was used to compare the data of means decay score in head, body and tail of the pancreas. $P$ value $<0.05$ taken as significant. Spearman correlation was used to compare the relation between decay score with time since death and immunoreactivity. Statistical test was performed by the using SPSS software version 25 and graphs were plotted by Microsoft Excel 2019 software.

\section{RESULTS}

The pancreatic samples were categorised into three groups based on the time since death: group 1 - less than 6 hours of death, group 2 - from 6 hours to 9 hours of the death and group 3 - more than 9 hours of death to 14 hours. 


\section{Micro-architectural changes in H\&E}

The decay score of all the individual pancreas was plotted in Figure 2 with its immunohistochemical reactivity status. Mean decay score of group 1, group 2 and group 3 are $1.351 \pm 0.715,1.409 \pm 0.225$ and $1.895 \pm 0.793$, respectively. The statistical significance was observed between group 1 and group 3 with a $p$-value of $<0.042$. The statistically significant positive correlation ( $r=0.479$ ) was observed between decay score and the time since death with the $p$-value of 0.033 . The mean decay score of head, body and tail of all the cases was $1.54 \pm 0.655,1.543 \pm 0.604$ and $1.56 \pm 0.645$. There was no statistically significant difference in the histological appearance of autolytic changes in various parts of the pancreas.

\section{Immunohistochemical reactivity}

Decay score and time since death were inversely proportional to the immunoreactivity of the pancreatic islets (Fig. 2). Strong immunoreactivity of pancreatic islets in groups 1, 2 and 3 was $80 \%, 72 \%$ and $42.85 \%$, respectively (Fig. 3 ). The mean decay score of the pancreatic islets with strong immunoreactivity was $1.22 \pm 0.182$ and $2.205 \pm 0.652$ for specimens with poor immunoreactivity. The statistically significant negative correlation ( $r=-0.736)$ was observed between the decay score and immune reactivity of pancreatic islets with the $p$-value of $<0.0001$. Furthermore, all the strong IHC positive tissue has decay score less than 1.4 except sample no. 20 which had weak immune reactivity and a decay score of 1.39 . Samples showing strong immunoreactivity for islets also shown similar reactivity for beta-cells.

\section{DISCUSSION}

Most of the studies on pancreatic islets have used animal models [1]. The studies involving microscopic analysis of human pancreatic islets are limited and the majority of these are from developed countries where tissue samples from the brain-dead donor are easily available $[7,11,16]$. The critical factor limiting the use of human pancreatic samples from the autopsy is the appearance of early autolytic changes in the pancreatic tissue (exocrine and endocrine) within an hour of death [13]. As the pancreas contains a high number of proteolytic enzymes, it undergoes rapid autodigestion soon after death. The autopsy done after 12 hours of death renders the pancreatic tissue unusable [13]. The various components of pancreatic tissue like exocrine, endocrine and stromal parts may

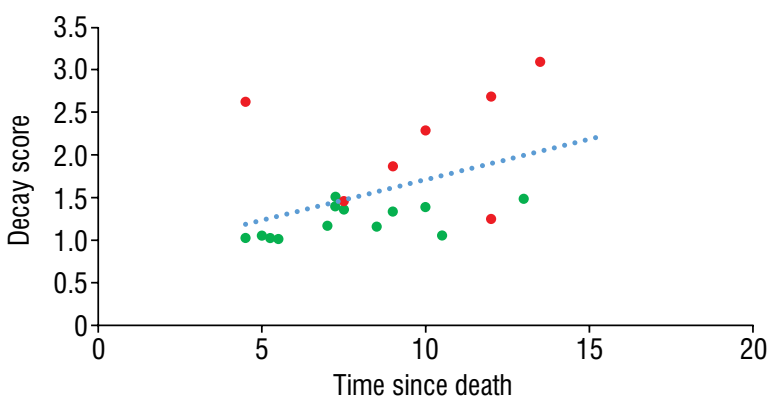

Figure 2. Scatter plot showing the comparison of decay score with time since death $\left(\mathrm{R}^{2}=0.185\right)$ and immunoreactivity status; red dot — poor immunoreactivity; green dot — good immunoreactivity

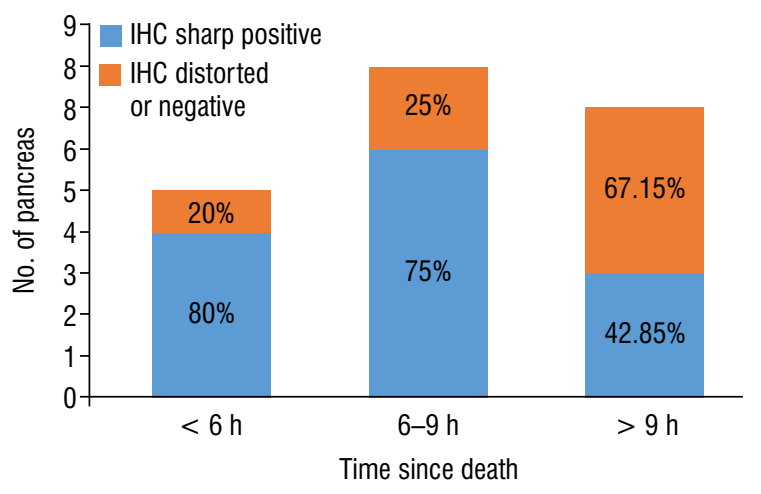

Figure 3. The percentage of pancreatic sample having preserved and diffused immunoreactivity in relation to time since death; $\mathrm{IHC}$ - immunohistochemical studies.

show a variable timeline of autolytic changes, i.e. the exocrine part undergoes autolysis earlier than endocrine and stromal components [4]. Siriwardana et al. [13], given the timeline of autolytic changes in the pancreas as a whole, which may not be useful for endocrine study. The current study is focused on the autolytic changes of endocrine pancreas (islets) to guide the IHC on pancreatic islets specifically.

As there is a paucity of data regarding time since death and an acceptable amount of autolytic changes in pancreatic islets, many studies in the literature have used a wide range of cut-off time varying from 6 hours to 24 hours after death or cold ischaemia. The present study shows that autolytic changes increase with time, which in turn inversely affects the immunoreactivity of the pancreatic islets. Authors found that $67.15 \%$ of pancreatic samples collected after 9 hours of death were showed diffused or absent immunoreactivity, but similar findings were found only in $23 \%$ of samples which were collected within 
9 hours of death. Thus, if the pancreatic tissue is preserved in formalin within 9 hours of death, its immunoreactivity is highly maintained ( $75 \%$ to $80 \%$ ). The finding of the present study is consistent with the existing literature in which the author graded the autolytic changes with H\&E staining without mentioning its immunoreactivity [5].

The autolytic changes of a particular islet will be heterogeneous in nature, i.e. all the cells in the islet may not show a similar grade of autolysis. The decay score system helps us to objectively score the autolytic changes in a particular islet. We observed an increasing trend of decay score with time. Higher the decay score of pancreatic islets represents the greater degree of tissue destruction, which in turn results in poor immunoreactivity of the tissue. We found that slides with a decay score of less than 1.4 had good immunoreactivity and this value (decay score 1.4) may be used as the cut off to proceed for IHC. Immunoreactivity of samples obtained from surgeries and the brain-dead donors were mainly influenced by the cold ischaemia time. The cold ischaemia time is the interval between the removal of tissue from the body and its contact with formalin. Autolytic changes still occur even in the period of cold ischaemia [5]. Autolytic changes may be delayed in the foetal pancreas (under 5-month) due to underdeveloped exocrine part (less proteolytic enzymes) when compared with adult pancreas [2].

Even though most of the cases showed a consistent relation between degree of autolysis and time since death; in 1 case severe autolytic changes (decay score of 2.63) was observed in less than 6 hours of death but in few other specimens well-preserved micro-architectures (decay score of 1.49) and staining immunogenicity were seen even after 12 hours of death. Similar findings have also been reported in post-mortem studies of lung, liver and brain tissue, in which authors justified that the above variation as a pattern of normal distribution [9]. Apart from that, the inaccurate record of the time of death in cases where the death occurred outside the hospital setup may explain the confounding results. In spite of the controversial observation between time since death and immunoreactivity, the relationship between the decay score and immunoreactivity of pancreatic tissue is maintained. Thus, the decay score might be used as a tool to know the antigenic status of the tissue prior to the immunohistochemistry of autopsy specimens.

Immunoreactivity is not only affected by time since death, but also by any defect in the tissue processing and staining procedure. Studies have reported that factors like fixation time, dehydration, clearing, paraffin impregnation, antigen retrieval and IHC staining procedures also play a significant role in the optimal expression of tissue antigenicity $[9,14]$. The authors believe that the factors involving tissue processing and staining have a very insignificant role in the present study as all the samples used here were processed and stained by using the standard protocol in the same laboratory setup.

\section{CONCLUSIONS}

Pancreatic islets immunoreactivity was found to be well preserved in the samples collected early within 9 hours with a decay score of less than 1.4. There is an inverse relationship between the decay score and immunoreactivity of islets, i.e. smaller the decay score, better the immunoreactivity. To the best of our knowledge, no literature is available on the autolytic changes and immunoreactivity of human pancreatic islets. The present study concludes that the time since death may not be the sole criterion to determine the immunoreactivity of the pancreatic islets. The decay score of pancreatic islets must be taken into consideration prior to the IHC. This knowledge will help the researchers working in the field of the endocrine pancreas.

\section{Funding/Support}

This work was supported in part by the Indian Council of Medical Research - Thesis grant no. 3/2/ /June-2017/PG-Thesis-HRD(14). The funding agency had no role in study design, data collection, analysis, interpretation and manuscript preparation.

\section{Conflict of interest: None declared}

\section{REFERENCES}

1. Abunasef SK, Amin HA, Abdel-Hamid GA. A histological and immunohistochemical study of beta cells in streptozotocin diabetic rats treated with caffeine. Folia Histochem Cytobiol. 2014; 52(1): 42-50, doi: 10.5603/ FHC.2014.0005, indexed in Pubmed: 24802960.

2. Banting FG, Best CH, Collip JB, et al. Pancreatic extracts in the treatment of diabetes mellitus. Can Med Assoc J. 1922; 12(3): 141-146, indexed in Pubmed: 20314060.

3. Campbell-Thompson ML, Montgomery EL, Foss RM, et al. Collection protocol for human pancreas. J Vis Exp. 2012(63): e4039, doi: 10.3791/4039, indexed in Pubmed: 22665046.

4. Cocariu EA, Mageriu V, Stăniceanu F, et al. Correlations between the autolytic changes and postmortem interval in refrigerated cadavers. Rom J Intern Med. 2016; 54(2): 
105-112, doi: 10.1515/rjim-2016-0012, indexed in Pubmed: 27352439.

5. Hilling DE, Bouwman E, Terpstra OT, et al. Effects of donor-, pancreas-, and isolation-related variables on human islet isolation outcome: a systematic review. Cell Transplant. 2014; 23(8): 921-928, doi: 10.3727/096368913X666412, indexed in Pubmed: 23635354.

6. Holtzer RL, Van Lancker JL. Early changes in pancreas autolysis. Am J Pathol. 1962; 40: 331-336, indexed in Pubmed: 13908608.

7. Ionescu-Tirgoviste C, Gagniuc PA, Gubceac E, et al. A 3D map of the islet routes throughout the healthy human pancreas. Sci Rep. 2015; 5: 14634, doi: 10.1038/ srep14634, indexed in Pubmed: 26417671.

8. Jones LC, Clark A. Beta-cell neogenesis in type 2 diabetes. Diabetes. 2001; 50 Suppl 1: S186-S187, doi: 10.2337/ diabetes.50.2007.s186, indexed in Pubmed: 11272189.

9. Lesnikova I, Schreckenbach MN, Kristensen MP, et al. Usability of Immunohistochemistry in Forensic Samples With Varying Decomposition. Am J Forensic Med Pathol. 2018; 39(3): 185-191, doi: 10.1097/PAF.0000000000000408, indexed in Pubmed: 29794805.

10. Olehnik SK, Fowler JL, Avramovich G, et al. Quantitative analysis of intra- and inter-individual variability of human beta-cell mass. Sci Rep. 2017; 7(1): 16398, doi: 10.1038/ s41598-017-16300-w, indexed in Pubmed: 29180621.

11. Poudel A, Fowler JL, Zielinski MC, et al. Stereological analyses of the whole human pancreas. Sci Rep. 2016;
6: 34049, doi: 10.1038/srep34049, indexed in Pubmed: 27658965.

12. Ravi PK, Purkait S, Agrawal U, et al. Regional variation of human pancreatic islets dimension and its impact on beta cells in Indian population. Islets. 2019; 11(6): 141-151, doi: 10.1080/19382014.2019.1686323, indexed in Pubmed: 31743072.

13. Siriwardana RC, Deen KI, Hevawesenthi J. Postmortem sampling of the pancreas for histological examination: what is the optimum cut-off time? JOP. 2010; 11(1): 87-88, indexed in Pubmed: 20065563.

14. Suvarna S, Layton C, Bancroft JD. Bancroft's Theory and Practice of Histology Techniques. 7th ed. Churchill Livingstone, China 2013.

15. Wang X, Misawa R, Zielinski MC, et al. Regional differences in islet distribution in the human pancreas - preferential beta-cell loss in the head region in patients with type 2 diabetes. PLoS One. 2013; 8(6): e67454, doi: 10.1371/ journal.pone.0067454, indexed in Pubmed: 23826303.

16. Wang X, Zielinski MC, Misawa R, et al. Quantitative analysis of pancreatic polypeptide cell distribution in the human pancreas. PLoS One. 2013; 8(1): e55501, doi: 10.1371/ journal.pone.0055501, indexed in Pubmed: 23383206.

17. Yagihashi S, Inaba W, Mizukami H. Dynamic pathology of islet endocrine cells in type 2 diabetes: beta-cell growth, death, regeneration and their clinical implications. J Diabetes Investig. 2016; 7(2): 155-165, doi: 10.1111/jdi.12424, indexed in Pubmed: 27042265. 